Extended Abstract

\title{
Sign and information: form and content
}

\author{
László Ropolyi \\ Department of History and Philosophy of Science, Eötvös University, H-1518 Budapest Pf. 32 \\ Hungary
}

E-Mail: ropolyi@caesar.elte.hu

Tel.: +36-1-372- 2949; Fax: +36-1-372-2924

Accepted:

\section{Introduction}

In this paper three closely related philosophical problems are discussed. Each of them can be considered as source of difficulties in understanding the very nature of information. First we focus on the ontological character of signs. Then a simple hermeneutic concept of information will be proposed. Finally an important dimension of the sign-information relationship will be introduced.

\section{On the ontological character of signs}

Considering the huge number of approaches to the concept of information and the numerous philosophical debates on it (see e.g. Capurro - Hjørland [1], Floridi [2], Hofkirchner [3]), it is not really surprising to entertain some confidence on a philosophical analysis of the ontological character of information.

In our view one of the most important ontological issues is the recognition that it is better to conceptualize the information as a relation instead of a thing. (If we are dialectical thinkers it is easy to see that every being is a thing, and at the same time it is a bunch of coexisting relations and at the same time it is a historical process.) Another fundamental philosophical issue is taking into seriously the sign-information differences and the sign-information relationship.

Even at first sight it is clear that the condition of the existence of information is the coexistence of two "different levels" of beings. One of the levels consists of beings/events, but at the same time, we can also identify beings/events existing on a different level which can correspond to the beings/events of the "first level". As a result of the correspondence, the beings/events of the "second level" become the signs of the beings/events of the first level (or of the relations between them). Signs have a crucial 
ontological character: they necessarily include and hold a relation, the relation between the beings/events of the above mentioned two different "levels of beings", or in a more general sense a relation between two (arbitrary) beings. A sign is an existing relation or relating existences (beings/events).

The constituents of an existing relation represent each other. A sign stands here and now instead of something else, refers to something else, "substituting" or representing that. A further understanding of the nature of representation has a fundamental role in the philosophy of information.

There is no representation without using signs. In other words: there is no representation without two kinds of beings, or two contexts for the beings. The sign has a specific, double nature: the sign is an actual being, but at the same time, potentially something else. We can identify something as a sign if and only if these two faculties of its nature (actually something and potentially something else) are simultaneously present.

In the above mentioned context: every kinds of "re-presentation" presupposes two kinds of beings (the beings what are represented and the beings what is representing that) or two different contexts for the beings (to consider the same thing in two different ways). It is the crucial that there is a necessary interrelationship between these two kinds of beings or contexts to create re-presentations. The representation based on the existence of this relation. The (free) creation of this relation sometimes called coding or signifying. Any kind of relation is a source of the "actually something, but potentially something else" nature of a thing. Representations produce necessarily - such - virtual beings. On the language of philosophy it can be stated that representations have a specific ontological characters: the ontology of relations, or interrelationships, which is the ontology of virtual beings. All beings produced by representational technologies are necessarily virtual.

\section{Hermeneutic concept of information - form and content}

In the history of culture a well known and widely accepted methodology can be found on the treatment and utilization of two interrelating beings (or two interrelating contexts for a being): hermeneutics. In hermeneutics the interpretation through which the correspondence of beings/events on different levels (or the correspondence of different contexts) can be established.

In this way it has a meaning to conceptualize signs as hermeneutical products, which are created through a direct mediation between two beings/events/contexts, shortly two "worlds".

However, if we want to conceptualize the information in a hermeneutic approach it is needed to repeat almost all again what was told above on the sign. Let us say in this way: in the process of the creation of information a mental act, interpretation, is needed, the active agent is without any doubt the interpreting man. His activity of interpretation consists in considering an event as a sign of another, that is, on the one hand he assumes that the chosen event includes the possibility of a sign, on the other, he interprets the realization of this possibility by developing a system of signs, for example. After a successful interpretation, he can infer the processes of the signified world, just "as if" he were trying to find out about them directly on their level. If we did not regard a sign as something which can be a sign of something, it would not be a sign. Furthermore, if we did not regard this possibility as something which is actually realized, it would not give us any information. It is obviously not enough to consider an abstract possibility, since that can be a sign of anything; that does not provide us with any knowledge. Thus, a sign used in the production of information is virtually the signified. 
From the earlier train of thoughts it can be clear that in hermeneutic approach signs and information are also created by interpretation. However, it would be necessary to make a clear distinction between the existence of an interpretation and the content of it. The existence of an interpretation is completely enough to identify something as a sign. A working interpretation, however, using the different contexts and interrelating to each other different beings/events produces also definite meanings for the signs. Signs in themselves have no meanings. Information is a meaningful sign. Summarily: information is an "interpreted being" - also the existence and the meaning of the interpretation. Based on meanings we can have acquaintance, knowledge, cognition, etc.

Based on these differences the sign-information relationship can be described using a form-content relationship. Sign is the form of the information, while meaning is the content of it. Information is a meaningful sign or a signified meaning - created by interpretation

\section{Conclusions}

The sign-information relationship has a close similarity with the relationship between formal and "dialectical" logic. Considering only the formal aspects of propositions we can build up more or less complete formal logical systems - but in many practical cases we are interested in the contents of propositions too, so we can try to create content-dependent logics as it was proposed several times by dialectical thinkers. Similarly, the formal aspects of information is extensively studied in numerous versions of information theories (it would probably be better to call them theories of signs), where the meanings are disregarded. In fact, the creation of some kind of content-dependent information science is not so popular and not so easy - similarly again to an effective dialectical logic.

\section{References}

1. Capurro, R.; Hjørland, B. The concept of information. In Annual Review of Information Science and Technology, Cronin, B., Eds.; American Society for Information Science and Technology: Bethesda, MD USA, 2003, Volume 37, pp. 343-411.

2. Floridi, L. The Philosophy of Information, OUP: Oxford, England, 2011.

3. Hofkirchner, W. ed.. The Quest for a Unified Theory of Information, Routledge: London, England, 1999.

(C) 2015 by the authors; licensee MDPI and ISIS. This abstract is distributed under the terms and conditions of the Creative Commons Attribution license. 